\title{
Clinical characteristics and genotypes in the ADVANCE baseline data set, a comprehensive cohort of US children and adolescents with Pompe disease
}

\author{
Priya S. Kishnani, MD ${ }^{1}{ }^{1}$, James B. Gibson, MD, PhD ${ }^{2}{ }^{2}$, Michael J. Gambello, $\mathrm{MD}^{3}$, \\ Richard Hillman, MD, David W. Stockton, MD, David Kronn, MD ${ }^{6}$, Nancy D. Leslie, MD ${ }^{7}$, \\ Loren D. M. Pena, MD, PhD ${ }^{1,7,8}$, Pranoot Tanpaiboon, MD ${ }^{9}$, John W. Day, MD, PhD ${ }^{10}$ \\ Raymond Y. Wang, MD ${ }^{11,12}$, Jennifer L. Goldstein, PhD, CGC ${ }^{1}$, Kristina An Haack, MD ${ }^{13}$, \\ Susan E. Sparks, MD, PhD ${ }^{13}$, Yang Zhao, $\mathrm{PhD}^{13}$ and \\ Si Houn Hahn, MD, $\mathrm{PhD}^{14}$ on behalf of the Pompe ADVANCE Study Consortium
}

\begin{abstract}
Purpose: To characterize clinical characteristics and genotypes of patients in the ADVANCE study of $4000 \mathrm{~L}$-scale alglucosidase alfa (NCT01526785), the largest prospective United States Pompe disease cohort to date.

Methods: Patients aged $\geq 1$ year with confirmed Pompe disease previously receiving $160 \mathrm{~L}$ alglucosidase alfa were eligible. GAA genotypes were determined before/at enrollment. Baseline assessments included histories/physical exams, Gross Motor Function Measure-88 (GMFM-88), pulmonary function tests, and cardiac assessments.

Results: Of 113 enrollees (60 male/53 female) aged 1-18 years, 87 had infantile-onset Pompe disease (IOPD) and 26 late-onset (LOPD). One hundred eight enrollees with GAA genotypes had 215 pathogenic variants (220 including combinations): 118 missense (4 combinations), 23 splice, 35 nonsense, 34 insertions/deletions, 9 duplications (1 combination), 6 other; c. $2560 \mathrm{C}>\mathrm{T}(n=23)$, c. $-32-13 \mathrm{~T}>\mathrm{G}(n=$ $13)$, and c.525delT $(n=12)$ were most common. Four patients had previously unpublished variants, and 14/83 (17\%) genotyped IOPD
\end{abstract}

patients were cross-reactive immunological material-negative. All IOPD and 6/26 LOPD patients had cardiac involvement, all without c. $-32-13 \mathrm{~T}>\mathrm{G}$. Thirty-two (26 IOPD, 6 LOPD) were invasively ventilated. GMFM- 88 total \%scores (mean $\pm \mathrm{SD}$, median, range): overall $46.3 \pm 33.0 \% \quad(47.9 \%, \quad 0.0-100.0 \%), \quad$ IOPD $41.6 \pm 31.64 \%$ (38.9\%, 0.0-99.7\%), LOPD: $61.8 \pm 33.2$ (70.9\%, 0.0-100.0\%).

Conclusion: ADVANCE, a uniformly assessed cohort comprising most US children and adolescents with treated Pompe disease, expands understanding of the phenotype and observed variants in the United States.

Genetics in Medicine (2019) 21:2543-2551; https://doi.org/10.1038/s41436019-0527-9

Keywords: alglucosidase alfa; glycogenosis type 2; GAA pathogenic variants; infantile-onset Pompe disease (IOPD); late-onset Pompe disease (LOPD)

\section{INTRODUCTION}

Pompe disease or glycogen storage disease type II (OMIM 232300) is a rare, autosomal recessive disorder of glycogen metabolism caused by deficiency of the glycogen-degrading lysosomal enzyme acid a-glucosidase (GAA, EC 3.2.1.20), leading to multisystemic lysosomal glycogen accumulation. Clinical symptoms primarily reflect cardiac, respiratory, skeletal, and smooth muscle involvement. ${ }^{1}$ The disease spectrum ranges from infantile-onset Pompe disease (IOPD; symptom onset $<12$ months of age with cardiomyopathy ${ }^{2}$ ) to the more slowly progressive late-onset Pompe disease (LOPD; symptom onset $<12$ months of age without cardiomyopathy or onset $\geq 12$ months of age ${ }^{2}$ ).

Prognosis for IOPD patients depends on several factors, including not only GAA genotypes (and any resulting residual functional GAA activity) but also cross-reactive immunologic material (CRIM) status. ${ }^{3}$ CRIM-negative patients harbor GAA variants (nonsense, insertion, deletion, or indel) abrogating native GAA protein synthesis or prematurely truncating the protein product, which is targeted for ubiquitination and

\footnotetext{
${ }^{1}$ Duke University Medical Center, Durham, NC, USA; ${ }^{2}$ Dell Children's Medical Group, Austin, TX, USA; ${ }^{3}$ Emory University School of Medicine, Atlanta, GA, USA; ${ }^{4}$ University of Missouri Child Health, Columbia, MO, USA; ${ }^{5}$ Children's Hospital of Michigan and Wayne State University, Detroit, MI, USA; ${ }^{6}$ New York Medical College, Valhalla, NY, USA; ${ }^{7}$ Cincinnati Children's Hospital, Cincinnati, OH, USA; ${ }^{8}$ University of Cincinnati College of Medicine, Cincinnati, OH, USA; ${ }^{9} \mathrm{Children}$ 's National Health System, Washington, DC, USA; ${ }^{10}$ Stanford University, Stanford, CA, USA; ${ }^{11}$ Children's Hospital of Orange County, Orange, CA, USA; ${ }^{12}$ University of California-Irvine School of Medicine, Irvine, CA, USA; ${ }^{13}$ Sanofi Genzyme, Cambridge, MA, USA; ${ }^{14}$ Seattle Children’s Hospital/University of Washington, Seattle, WA, USA. Correspondence: Priya S. Kishnani (priya.kishnani@duke.edu) Affiliations when the study was conducted: Loren D. M. Pena ${ }^{1}$, Pranoot Tanpaiboon ${ }^{9}$, Jennifer L. Goldstein ${ }^{1}$ and Yang Zhao ${ }^{13}$
} 
protealyzed. ${ }^{4}$ In contrast, CRIM-positive patients produce some immunologically recognizable GAA protein. CRIM status can usually be predicted from genotypes. ${ }^{3}$ The degree of Pompe disease progression at treatment initiation strongly affects prognosis. Diagnostic delays, particularly in IOPD, lead to worse outcomes. ${ }^{2}$

Alglucosidase alfa (recombinant human GAA [rhGAA]) received regulatory approval in 2006 in the United States and Europe as the first and only disease-specific treatment for Pompe disease. The studies supporting initial approval demonstrated increased overall and ventilator-free survival and cardiac, respiratory, and motor improvement in IOPD patients receiving alglucosidase alfa produced at a $160 \mathrm{~L}$ pilot scale (160 L rhGAA). ${ }^{5,6}$ Alglucosidase alfa manufactured at a larger scale (Lumizyme $^{\circledR}$ ) was evaluated in LOPD patients $\geq 8$ years of age without cardiomyopathy, ${ }^{7}$ receiving US approval in 2010 initially for that population. The ADVANCE study (NCT01526785) evaluated comparability of $4000 \mathrm{~L}$ rhGAA safety and efficacy in pediatric $160 \mathrm{~L}$ rhGAA-experienced patients with Pompe disease, irrespective of cardiac phenotype. ${ }^{8}$ Its safety data supported the US label expansion of Lumizyme $^{\circledR}$ (alglucosidase alfa) in 2014 to include all patients with Pompe disease.

The ADVANCE study was open to US patients with a confirmed Pompe disease diagnosis (deficient endogenous GAA activity and/or GAA genotype) $\geq 1$ year of age at study entry who were receiving $160 \mathrm{~L}$ rhGAA at enrollment. This contemporaneous, prospective cohort of 113 patients provides a unique, one-time opportunity to understand the genotypic and phenotypic spectrum of Pompe disease in the United States via systematic clinical histories and assessments, to compare variant types found in IOPD and LOPD, and to ascertain novel Pompe disease variants within the population.

\section{Design and ethics}

MATERIALS AND METHODS

This prospective, open-label, single-arm study was conducted at 52 centers in 30 states plus Washington, DC (29 March 2012-29 December 2014). Parents/guardians (or patients if $\geq 18$ years old) gave written informed consent; children assented whenever possible. Western Institutional Review Board (IRB; Puyallup, WA) or local sites' IRBs (online appendix) gave ethical approval; Good Clinical Practice, the Declaration of Helsinki 1964 and applicable amendments ${ }^{9}$, and all applicable laws, regulations, and guidelines were followed.

\section{Patients}

Patients aged $\geq 1$ year with enzymatically and/or molecularly confirmed Pompe disease previously treated with $160 \mathrm{~L}$ rhGAA were eligible. Patients enrolled in other studies or receiving other investigational treatments within 3 months before screening, unlikely to survive through 52 weeks of treatment, or unable to comply with study procedures were excluded. Negative pregnancy tests were required for postmenarchal female patients.

\section{Assessments}

GAA genotypes (reference sequence NM_000152) were determined at or before screening (five enzymatically diagnosed patients lacked genetic data). Prior GAA genotypes determined before study entry were used if provided by a certified laboratory with guardians'/patients' written informed consent to share with the site. In the case of missing genotype information, informed consent was obtained to conduct nextgeneration sequencing (NGS) of the GAA gene by LabCorp (www.LabCorp.com) (GAA Inheritest $\left.{ }^{\circledR}, 451910\right)$ and share the data with the site. Prediction of IOPD patients' CRIM status relied on historical data from identical $G A A$ variants identified in other patients. Patients whose $G A A$ variants precluded GAA protein production were considered CRIMnegative. ${ }^{3}$ Reported variants used in CRIM prediction were presumed to be (1) those causing Pompe disease (supported by database or literature reports of pathogenicity and/or by GAA enzyme activity levels), i.e., patients did not have another, unidentified pathogenic variant; and (2) in trans (reported in-cis combinations were confirmed by parental testing).

Left ventricular mass (LVM) $z$-scores were determined via locally read and centrally reviewed M-mode echocardiography. Standard 12-lead electrocardiography, locally read and reviewed by a central cardiologist blinded to patient and study timepoint, ascertained left or right ventricular hypertrophy criteria and heart rhythm. Congestive heart failure (CHF) was determined clinically, with New York Heart Association (NYHA) staging.

Invasive or noninvasive ventilator dependence was scored as of the first ventilation date not followed by 14 consecutive ventilator-independent days. For testable ventilator-free patients, seated (upright) forced vital capacity (FVC) \% predicted was determined following American Thoracic Society guidelines.

Motor skills were assessed via Gross Motor Function Measure, 88 items (GMFM-88), which has precedent (though not formal validation) for use in Pompe disease. ${ }^{10,11}$

Motor function levels and histories were assessed at study entry only, classifying patients as walkers, supported walkers, supported standers, sitters, or restricted antigravity movement. Questions on motor decline during the 2 months before enrollment were specific to functional level and age group $(<2$ or $\geq 2$ years of age).

Medical histories were collected emphasizing systems affected by Pompe disease. Biochemical testing included serum creatine kinase, brain natriuretic peptide, and aspartate and alanine aminotransferases.

\section{Analyses}

Demographic, clinical, and genetic characteristics are reported as summary statistics overall and (post hoc) for IOPD and LOPD subsets. IOPD patients were those with symptom onset 
by 12 months of age and evidence of cardiac involvement (cardiomegaly, cardiomyopathy, or hypertrophies), potentially including some patients with less severe cardiac phenotypes.

\section{RESULTS}

Demographics, clinical characteristics, and medical histories One hundred thirteen patients were enrolled (aged 1.0-18.7 years at consent; Table 1). The CONSORT diagram was published previously. ${ }^{8}$ The one excluded patient had previously received commercial $4000 \mathrm{~L}$ rhGAA. At consent, ages of IOPD patients were 1.0-15.5 years (mean \pm SD, $4.4 \pm$ 3.52 years; median, 3.4 years); those of LOPD patients were 1.1-18.7 years (mean $\pm \mathrm{SD}, 6.0 \pm 4.19$ years; median, 6.1 years). IOPD was defined as symptom onset at $<12$ months of age with cardiac involvement, and LOPD as symptom onset either at $<12$ months of age without cardiac involvement or at $\geq 12$ months of age. ${ }^{2}$ Eighty-seven patients had IOPD and 26 had LOPD (20 without and 6 with cardiac involvement).

Of the 87 IOPD patients, 70 had symptom onsets and $160 \mathrm{~L}$ rhGAA initiations before 12 months of age. Seventeen more met IOPD criteria but had survived $>12$ months before $160 \mathrm{~L}$ rhGAA initiation and could include advanced and less severe IOPD cases. Their reported age range at symptom onset was 0-8.8 months, at diagnosis 40 days-5.9 years. Reported $160 \mathrm{~L}$ rhGAA initiations were at 1.0 to $\sim 11$ years, and ages at ADVANCE enrollment 2.1-15.5 years. Two of these had received other investigational enzyme therapies before $160 \mathrm{~L}$ initiation (one received Synpac rhGAA starting at $\sim 2$ years of age, then $160 \mathrm{~L}$ rhGAA at $\sim 11$ years of age; the other received expanded-access rhGAA starting at $\sim 6.9$ years of age, then $160 \mathrm{~L}$ rhGAA at $\sim 7.9$ years of age). Further details are in ADVANCE efficacy publication supplementary materials. ${ }^{8}$ All IOPD patients were analyzed together.

LOPD symptom onset ranged from 1.1 to 5 years in the 6 patients with cardiomyopathy/cardiomegaly, versus birth-8.9 years in the other 20 patients. Proportionately more LOPD than IOPD patients were Caucasian ( $88 \%$ vs. $55 \%)$, male $(73 \%$ vs. $47 \%)$, ventilator-free $(69 \%$ vs. $59 \%)$, and walked independently $(27 \%$ vs. $18 \%)$ or with assistance $(35 \%$ vs. $18 \%)$. Only $8 \%$ of LOPD patients, but $28 \%$ of IOPD patients, had restricted antigravity movement at enrollment (Table 1).

Thirty-two patients were invasive ventilator-dependent (26 IOPD, 6 LOPD) for a median of 3.9 years (mean \pm SD, $4.8 \pm$ 4.14 years; range, $0.1-17.0$ years). Eleven further patients were noninvasive ventilator-dependent (9 IOPD, 2 LOPD). Seventy-five $(66 \%)$ patients had histories of pneumonia (61/ 87 [70\%] with IOPD and 14/26 [54\%] with LOPD).

Motor symptomatology at study entry (Table 2) included lower extremity weakness in 101 (89\%) patients, upper extremity weakness in $94(83 \%)$, and motor milestone regression in 45 (40\%). At screening, 48 (42\%) patients were ambulatory (independently or device-assisted); 65 (58\%) were nonambulatory at screening, 11 of whom had ever walked. In all, $54(48 \%)$ used ambulatory or mobility devices. Most never-ambulatory patients had later $160 \mathrm{~L}$ initiations (35
Table 1 Baseline demographics and summary cardiac, pulmonary, motor, and growth parameters (full analysis set and disease state subsets)

\begin{tabular}{|c|c|c|c|}
\hline Parameter & Total $(N=113)$ & IOPD $(n=87)$ & $\operatorname{LOPD}(n=26)$ \\
\hline \multicolumn{4}{|l|}{ Sex, $n(\%)$} \\
\hline Male & $60(53)$ & $41(47)$ & $19(73)$ \\
\hline Female & $53(47)$ & $46(53)$ & $7(27)$ \\
\hline \multicolumn{4}{|l|}{ Ethnicity, $n$ (\%) } \\
\hline Hispanic & $18(16)$ & $15(17)$ & $3(12)$ \\
\hline Non-Hispanic & $92(81)$ & $70(81)$ & $22(85)$ \\
\hline Unreported & $1(1)$ & 0 & $1(4)$ \\
\hline Unknown & $2(2)$ & $2(2)$ & 0 \\
\hline \multicolumn{4}{|l|}{ Race, $n(\%)$} \\
\hline Asian & $7(6)$ & $7(8)$ & 0 \\
\hline African American & $26(23)$ & $24(28)$ & $2(8)$ \\
\hline Caucasian & $71(63)$ & $48(55)$ & $23(89)$ \\
\hline Unreported & $2(2)$ & $2(2)$ & 0 \\
\hline Multiple & $7(6)$ & $6(7)$ & $1(4)$ \\
\hline $\begin{array}{l}\text { Age at first } \\
\text { symptoms, mean } \pm \\
\text { SD (median, range; } \\
n \text { ) }\end{array}$ & $\begin{array}{l}0.5 \pm 1.15(0.3 \\
0.0-8.9 ; 112)\end{array}$ & $\begin{array}{l}0.2 \pm 0.22(0.2 \\
0.0-0.7 ; 87)\end{array}$ & $\begin{array}{l}1.5 \pm 2.08 \\
(0.9 \\
0.0-8.9 ; 26)\end{array}$ \\
\hline $\begin{array}{l}\text { Age at initial } \\
\text { diagnosis, years, } \\
\text { mean } \pm \text { SD } \\
\text { (median, range; } n \text { ) } \\
\text { Ambulatory at enroll }\end{array}$ & $\begin{array}{l}0.9 \pm 1.40(0.5 \\
0.0-8.8 ; 113)\end{array}$ & $\begin{array}{l}0.5 \pm 0.73(0.4 \\
0.0-5.9 ; 87)\end{array}$ & $\begin{array}{l}2.3 \pm 2.12 \\
(1.5 \\
0.0-8.8 ; 26)\end{array}$ \\
\hline Yes & $48(43)$ & $33(38)$ & $15(58)$ \\
\hline No & $65(58)$ & $54(62)$ & $11(42)$ \\
\hline $\begin{array}{l}\text { If no, ever } \\
\text { ambulatory, } n(\%)\end{array}$ & $11(10)$ & $10(12)$ & $1(4)$ \\
\hline $\begin{array}{l}\text { Using ambulatory } \\
\text { devices at } \\
\text { enrollment, } n(\%)\end{array}$ & $54(48)$ & $43(49)$ & $11(42)$ \\
\hline Walker & $10(9)$ & $7(8)$ & $3(12)$ \\
\hline Wheelchair & 39 (35) & $32(37)$ & $7(27)$ \\
\hline Other & $23(20)$ & $16(18)$ & $7(27)$ \\
\hline $\begin{array}{l}\text { Using ventilatory } \\
\text { support at } \\
\text { enrollment, } n(\%)\end{array}$ & $43(38)$ & $35(40)$ & $8(31)$ \\
\hline Invasive & $32(28)$ & $26(30)$ & $6(23)$ \\
\hline Noninvasive & $11(10)$ & $9(10)$ & $2(8)$ \\
\hline None & $70(62)$ & $52(60)$ & $18(69)$ \\
\hline \multicolumn{4}{|c|}{ Pompe disease motor function categories, $n(\%)$} \\
\hline Walking & $23(20)$ & $16(18)$ & $7(27)$ \\
\hline $\begin{array}{l}\text { Supported } \\
\text { walking }\end{array}$ & $25(22)$ & $16(18)$ & $9(35)$ \\
\hline $\begin{array}{l}\text { Supported } \\
\text { standing }\end{array}$ & $15(13)$ & $12(14)$ & $3(12)$ \\
\hline Sitting & $21(19)$ & $16(18)$ & $5(19)$ \\
\hline $\begin{array}{l}\text { Restricted } \\
\text { antigravity } \\
\text { movement }\end{array}$ & $26(23)$ & $24(28)$ & $2(8)$ \\
\hline $\begin{array}{l}\text { Height } z \text {-score, } \\
\text { mean } \pm \text { SD } \\
(\text { median, range; } n)^{a}\end{array}$ & $\begin{array}{l}-0.04 \pm 1.457 \\
(0.02,-5.49 \text { to } \\
3.28 ; 106)\end{array}$ & $\begin{array}{l}-0.04 \pm 1.574 \\
(-0.02,-5.49 \\
\text { to } 3.28 ; 80)\end{array}$ & $\begin{array}{l}-0.06 \pm 1.042 \\
(-2.07 \text { to } \\
1.49 ; 26)\end{array}$ \\
\hline $\begin{array}{l}\text { Weight } z \text {-score, } \\
\text { mean } \pm \text { SD } \\
(\text { median, range; } n)^{\mathrm{a}}\end{array}$ & $\begin{array}{l}-0.20 \pm 1.210 \\
(-0.09,-4.30 \\
\text { to } 2.46 ; 111)\end{array}$ & $\begin{array}{l}-0.22 \pm 1.245 \\
(-0.09,-4.30 \\
\text { to } 2.46 ; 85)\end{array}$ & $\begin{array}{l}-0.12 \pm 1.105 \\
(-0.07 \\
-2.08 \\
2.40 ; 26)\end{array}$ \\
\hline $\begin{array}{l}\text { Combined weight } \\
\text { for length/stature } \\
z \text {-score, mean } \pm S D \\
(\text { median, range; } n)^{a}\end{array}$ & $\begin{array}{l}-0.25 \pm 1.357 \\
(-0.07 ;-5.05 \\
\text { to } 2.85 ; 77)\end{array}$ & $\begin{array}{l}-0.21 \pm 1.327 \\
(-0.01 ;-5.05 \\
\text { to } 2.14 ; 62)\end{array}$ & $\begin{array}{l}-0.44 \pm 1.509 \\
(-0.42,-2.66 \\
\text { to } 2.85 ; 15)\end{array}$ \\
\hline $\begin{array}{l}\text { LVM } z \text {-score (M- } \\
\text { mode); mean } \pm \text { SD } \\
\text { (median, range; } n \text { ) }\end{array}$ & $\begin{array}{l}-0.1 \pm 2.63 \\
(-0.6,-4.0 \text { to } \\
10.3 ; 85)\end{array}$ & $\begin{array}{l}0.4 \pm 2.80 \\
(-0.6,-3.8 \text { to } \\
10.3 ; 62)\end{array}$ & $\begin{array}{l}-1.5 \pm 1.42 \\
(-1.4,-4.0 \text { to } \\
1.0 ; 23)\end{array}$ \\
\hline $\begin{array}{l}\text { Upright (seated) } \\
\text { FVC \% predicted; } \\
\text { mean } \pm \text { SD } \\
(\text { median, range; } n)\end{array}$ & $\begin{array}{l}66.6 \pm 29.59 \\
(64.5, \\
22.0-116.0 ; 24)\end{array}$ & $\begin{array}{l}55.9 \pm 26.74 \\
(49.0, \\
22.0-113.0 ; 15)\end{array}$ & $\begin{array}{l}84.4 \pm 26.34 \\
(94.0, \\
34.0-116.0 ; 9)\end{array}$ \\
\hline $\begin{array}{l}\text { GMFM-88 total \% } \\
\text { score; mean } \pm \text { SD } \\
\text { (median, range; } n \text { ) }\end{array}$ & $\begin{array}{l}46.3 \pm 32.97 \\
(47.9 \\
0.0-100.0 ; 108)\end{array}$ & $\begin{array}{l}41.6 \pm 31.64 \\
(38.9, \\
0.0-100.0 ; 83)\end{array}$ & $\begin{array}{l}61.8 \pm 33.16 \\
(70.9 \\
0.0-100.0 ; 25)\end{array}$ \\
\hline
\end{tabular}

FVC forced vital capacity, GMFM-88 Gross Motor Function Measure 88 items, IOPD infantile-onset Pompe disease, LOPD late-onset Pompe disease, LVM left ventricular mass.

${ }^{a}$ Growth parameter $z$-scores for patients $0-2$ years of age were calculated via http://peditools.org/growthwho/index.php; those for patients 2-20 years of age, via http://peditools.org/growthpedi/index.php. 
Table 2 Pompe disease history at study entry (full analysis set and disease state subsets)

\begin{tabular}{|c|c|c|c|}
\hline Parameter & Total, $n(\%) N=113$ & IOPD, $n(\%) n=87$ & LOPD, $n(\%) n=26$ \\
\hline Sibling with confirmed Pompe disease & $19(17)$ & $13(15)$ & $6(23)$ \\
\hline Evidence of cardiac involvement & $93(82)$ & $87(100)$ & $6(23)$ \\
\hline Tracheostomy ever performed & $32(28)$ & $26(30)$ & $6(23)$ \\
\hline History of pneumonia & $75(66)$ & $61(70)$ & $14(54)$ \\
\hline \multicolumn{4}{|c|}{ Number of episodes in the past year at screening, $n(\%)$ of those reporting pneumonias } \\
\hline$<5$ & $73(97)$ & $60(98)$ & $13(93)$ \\
\hline $5-10$ & $2(3)$ & $1(2)$ & $1(7)$ \\
\hline Sleep disturbances & $39(35)$ & $32(37)$ & $7(27)$ \\
\hline Sleep apnea & $28(25)$ & $21(24)$ & $7(27)$ \\
\hline Lower extremity muscle weakness & $101(89)$ & $77(89)$ & $24(92)$ \\
\hline Upper extremity muscle weakness & $94(83)$ & $72(83)$ & $22(85)$ \\
\hline Regression of motor milestones & $45(40)$ & $34(39)$ & $13(50)$ \\
\hline Hypotonia & $108(96)$ & $83(95)$ & $25(96)$ \\
\hline Scoliosis & $25(22)$ & $19(22)$ & $6(23)$ \\
\hline \multicolumn{4}{|c|}{ If scoliosis reported, $n(\%)$ of those reporting scoliosis } \\
\hline Surgical interventions for scoliosis & $3(12)$ & $2(11)$ & $1(2)$ \\
\hline Using orthopedic devices for scoliosis & $9(36)$ & $7(37)$ & $2(3)$ \\
\hline Joint contractures & $45(40)$ & $36(41)$ & $9(35)$ \\
\hline Surgical repair of joint contractures & $9(8)$ & $7(8.0)$ & $2(8)$ \\
\hline Using orthopedic devices to treat contractures & $23(20)$ & $19(22)$ & $4(15)$ \\
\hline Failure to thrive & $50(44)$ & $39(45)$ & $3(12)$ \\
\hline Feeding difficulties & $78(69)$ & $65(75)$ & $13(50)$ \\
\hline Gastroesophageal reflux & $56(50)$ & $48(55)$ & $8(31)$ \\
\hline \multicolumn{4}{|l|}{ Clinical presentation, $n(\%)$ of those reporting reflux } \\
\hline $\mathrm{pH}$ probe & $4(7)$ & $3(0.06)$ & $1(13)$ \\
\hline Barium swallow & $17(30)$ & $16(33)$ & $1(13)$ \\
\hline Other & $15(27)$ & $12(25)$ & $3(38)$ \\
\hline On nutritional support at enrollment & $71(63)$ & $54(62)$ & $17(65)$ \\
\hline \multicolumn{4}{|c|}{ Type of support, if on nutritional support, $n(\%)$ of those on nutritional support } \\
\hline Tube feeding & $52(73)$ & $45(83)$ & $7(41)$ \\
\hline High caloric diet & $6(9)$ & $3(6)$ & $3(18)$ \\
\hline Parenteral nutrition & $0(0)$ & $0(0)$ & $0(0)$ \\
\hline High protein diet & $6(9)$ & $3(6)$ & $3(18)$ \\
\hline Other & $5(7)$ & $3(6)$ & $2(12)$ \\
\hline Tympanic membrane tubes placed & $32(28)$ & $26(30)$ & $6(23)$ \\
\hline \multicolumn{4}{|l|}{ Hearing loss at enrollment } \\
\hline Yes & $33(29)$ & $28(32)$ & $5(19)$ \\
\hline No & $74(67)$ & $53(61)$ & $21(81)$ \\
\hline Assessment not done & $6(5)$ & $6(7)$ & $0(0)$ \\
\hline \multicolumn{4}{|c|}{ Location of hearing loss if present, $n(\%)$ of those with hearing loss } \\
\hline Unilateral & $3(9)$ & $2(7)$ & $1(20)$ \\
\hline Bilateral & $28(85)$ & $24(86)$ & $4(80)$ \\
\hline \multicolumn{4}{|c|}{ Type of hearing loss: left ear, $n(\%)$ of those with hearing loss } \\
\hline Sensorineural & $7(21)$ & $6(21)$ & $1(20)$ \\
\hline Conductive & $15(46)$ & $13(46)$ & $2(40)$ \\
\hline Mixed & $1(3)$ & $1(4)$ & $0(0)$ \\
\hline Unknown & $9(27)$ & $7(25)$ & $2(40)$ \\
\hline Not applicable & $2(6)$ & $2(7)$ & $0(0)$ \\
\hline \multicolumn{4}{|c|}{ Type of hearing loss: right ear, $n(\%)$ of those with hearing loss } \\
\hline Sensorineural & $7(21)$ & $6(21)$ & $1(20)$ \\
\hline Conductive & $14(42)$ & $12(43)$ & $2(40)$ \\
\hline Mixed & $1(3)$ & $1(4)$ & $0(0)$ \\
\hline Unknown & $7(21)$ & $6(21)$ & $1(20)$ \\
\hline Not applicable & $5(15)$ & $4(14)$ & $1(20)$ \\
\hline
\end{tabular}

IOPD infantile-onset Pompe disease, $L O P D$ late-onset Pompe disease.

patients initiated at $>6$ months of age versus 10 initiated at 3-6 months and 9 initiated at 0-3 months).

Sleep disturbance/apnea, failure to thrive, gastric reflux, chronic otitis media, and hearing loss appeared more prevalent in IOPD than LOPD (Table 2). Conductive hearing loss affected 13/87 (15\%) IOPD and 2/26 (8\%) LOPD patients; sensorineural hearing loss affected 6/87 (7\%) IOPD and $1 / 26(4 \%)$ LOPD patients.

\section{Family histories}

Nineteen (17\%) patients had a sibling with Pompe disease. Five phenotypically concordant sibling pairs (three pairs with IOPD and two pairs with LOPD) jointly participated in
ADVANCE; 8 patients had nonparticipating affected siblings, and 1 LOPD patient without genetic data had an affected sibling. One IOPD patient's mother had an LOPD genotype (11 patients' parents were tested).

\section{GAA pathogenic variants}

In the full analysis set $(n=113), 108(96 \%)$ patients had genetic data comprising 215 GAA pathogenic variants (220 including in-cis combinations) (Fig. 1; Supplementary Table S1). Four IOPD patients and one LOPD patient had missing genotypes. Two patients had three variants, two in cis and one in trans, and one patient had four variants: two in-cis combinations in trans to each other. The three most frequent 
in the IOPD cohort were nonsense c.2560C $>\mathrm{T}$, historically prevalent among African Americans ${ }^{12}$ (p.R854* 21 alleles), deletion c.525 $\operatorname{delT}^{13}$ (p.E176fs ${ }^{\star} 45$, nine alleles), and missense c.2297A $>C^{14}$ (p.Y766S, six alleles). Those most frequent in the LOPD cohort were c. $-32-13 \mathrm{~T}>\mathrm{G}^{15}$ (r.spl, 13 alleles), deletion c.525delT ${ }^{13}$ (p.E176fs ${ }^{\star} 45$, three alleles), and missense c.1210G $>\mathrm{A}^{16}$ (p.D404N, two alleles). The three most frequent overall were nonsense c.2560C $>T^{12}$ (p.R854 ; 23 alleles, 2 in LOPD and 21 in IOPD), splice variant c. -32 $-13 \mathrm{~T}>\mathrm{G}^{15}$ (13 alleles in LOPD), and deletion c.525delT ${ }^{13}$ (p. E176fs ${ }^{\star} 45 ; 12$ alleles, 9 in IOPD and 3 in LOPD). Most patients with c.2560C $>\mathrm{T}$ were African American: all 3 homozygotes with IOPD, 11 of 15 compound heterozygotes with IOPD (others: 2 multiracial and 2 Caucasian), and 1 of 2 compound heterozygotes with LOPD (the other was multiracial).

Unpublished variants occurred in four patients (Supplementary Table S2), by comparisons as of 8 March 2018 with current Erasmus $^{17}$ and Duke ${ }^{18}$ Pompe variant databases, ClinVar, and PubMed. In addition to three unpublished in-cis variant combinations shown in Supplementary Table S2 (see Supplementary Table S1 for references of their component variants), published in-cis combination c. [1642G $>\mathrm{T}$; $1880 \mathrm{C}>\mathrm{T}]]^{16}$ occurred in one IOPD patient.

Missense variant c.1844G>A (p.G615Q), homozygous in two IOPD patients, appeared only in the Duke database.

The unpublished splice variant c. $-32-17_{-}-32$ -10delinsTCCCCTGCTGAGCCTCCTACAGGCCTCCCGC in one LOPD patient was similar to a published variant ${ }^{19}$ but with one more repeating $\mathrm{C}$.

Exon 18 deletion c. $2481+102 \_2648+31$ del $^{20}$ was compound heterozygous in six patients (five IOPD, one LOPD with cardiac involvement). Two additional LOPD patients were compound heterozygous for exon 18 deletions with different breakpoints (c.2481+120_2646 and c.1799G >A with symptom onset $>12$ months of age and cardiomegaly; c.2481 +109_2848+38del538 and c.1979G $>$ A without cardiac involvement), which were not regarded as unpublished variants because the underlying large exon 18 deletion is the same.

c. $307 \mathrm{~T}>\mathrm{G}$ (p.C103G), common in Germany, ${ }^{21}$ occurred in compound heterozygosity in three patients with IOPD and one with LOPD (all Caucasian). Three Asian American patients had common Asian variants. c.2238G $>$ C (p.W746C), frequent in Chinese patients with LOPD, ${ }^{22}$ occurred in one IOPD patient (compound heterozygous with c.1843G $>$ A). c.1935C >A, a founder effect Chinese IOPD variant, ${ }^{22}$ occurred in two IOPD patients, one homozygous and one compound heterozygous with c.2815_2816delGT.

The common splice variant c. $-32-13 \mathrm{~T}>\mathrm{G}^{15}$ occurred in 12/20 LOPD patients (homozygous in 1 patient and compound heterozygous in 11; Supplementary Table S3). None of these 12 patients had any cardiomyopathy. Symptom onset occurred at 107 months of age in the c. $-32-13 \mathrm{~T}>\mathrm{G}$ homozygote, at 0-48 months (median, 5.9 months) in c. -32 $-13 \mathrm{~T}>\mathrm{G}$ compound heterozygotes, and at $0-60$ months (median, 20 months) in non-c. $-32-13 \mathrm{~T}>\mathrm{G}$ LOPD patients.
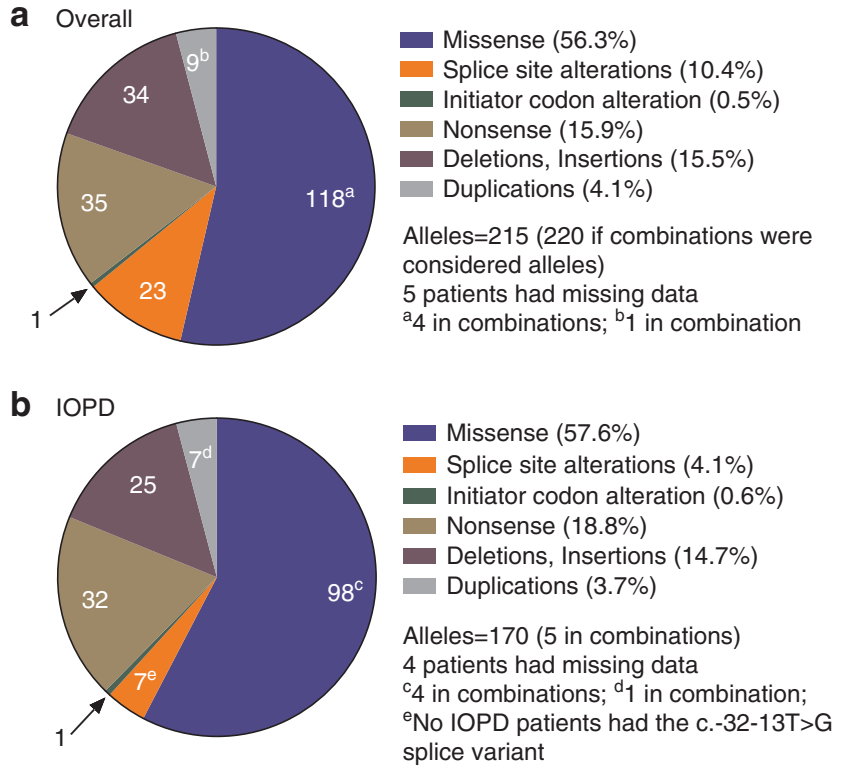

c LOPD

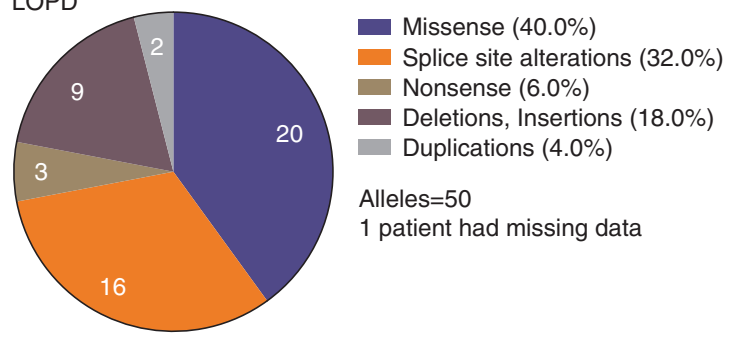

Fig. 1 GAA pathogenic variant types in ADVANCE. Distributions of GAA pathogenic variant types in the ADVANCE cohort (a) and its infantileonset Pompe disease (IOPD) (b) and late-onset Pompe disease (LOPD) (c) subsets. No patients with IOPD (nor with cardiac involvement in LOPD) had the common LOPD-associated splice variant c. $-32-13 T>G$.

All six LOPD patients with cardiac involvement (Supplementary Table S4) lacked c. $-32-13 \mathrm{~T}>\mathrm{G}$.

The severe splice c. $2331+21 \mathrm{~T}>\mathrm{A}^{23}$ and nonsense c. $1548 \mathrm{G}>\mathrm{A}^{24}$ variants were compound heterozygous with less severe c.670C $>\mathrm{T}$ in cardiac-involved LOPD patients. ${ }^{25}$ Less severe variants in cardiac-involved LOPD patients were c. $871 \mathrm{C}>\mathrm{T}^{26}$ (compound heterozygous with an exon 18 deletion), c.1445G $>\mathrm{A}^{27}$ (compound heterozygous with c. $1210 \mathrm{G}>\mathrm{A},{ }^{16}$ twins), and $\mathrm{c} .1799 \mathrm{G}>\mathrm{A}^{28}$ (compound heterozygous with an exon 18 deletion).

\section{CRIM status predictions}

Supplementary Table S5 lists IOPD genotypes, CRIM statuses, initial anti-alglucosidase alfa antibody titers, and cardiac phenotypes. Sixty-eight of 83 (82\%) genotyped IOPD patients were predicted CRIM-positive. Fifty-one had $\geq 1$ CRIMpositive variant identifiable from prior fibroblast studies, and the other 17 were predicted post hoc from genotypes. Fourteen (17\%) had CRIM-negative variants confirmed by prior fibroblast studies. Homozygous c.925G $>$ A (p.G309R) has unclear CRIM status, with conflicting literature reports. ${ }^{29,30}$ CRIM could not be predicted for one patient 
with an incompletely reported genotype or for four IOPD patients without genotypes.

\section{Shared genotypes}

Supplementary Table S3 shows all reported genotypes in IOPD and LOPD, their shared or unshared status within ADVANCE, and whether patients had affected siblings. No complete genotypes were shared between ADVANCE IOPD and LOPD patients.

In IOPD, two siblings shared splice variant c. $-32-17 \_-32$ -10 del8ins $30^{31}$ and missense variant c.1979G $>A^{32}$ Two other siblings shared nonsense c.525delT ${ }^{13}$ and missense c. $1448 \mathrm{G}>\mathrm{T}^{23}$ A third sibling pair shared splice c.546+2_ +5 delTGGG $^{3}$ and duplication c. 1650 dupG. $^{3}$ Three unrelated patients were homozygous for missense variant c.2297A $>C$. ${ }^{14}$ Homozygous missense c. $1843 \mathrm{G}>\mathrm{A}^{28}$ was shared by two unrelated patients, as was homozygous missense c.1844G $>$ A. ${ }^{18}$ Seven unrelated patients shared genotypes containing nonsense variant c. $2560 \mathrm{C}>\mathrm{T}^{12}$ : three were homozygous (all African Americans). Two unrelated African American patients were compound heterozygous with c. $1447 \mathrm{G}>\mathrm{A}$ as the second pathogenic variant. ${ }^{23}$ One Caucasian and one African American were compound heterozygous with c.525delT as the second pathogenic variant. ${ }^{13}$

In LOPD, twins with cardiac involvement shared c. $1210 \mathrm{G}>\mathrm{A}^{16}$ and $\mathrm{c} .1445 \mathrm{C}>\mathrm{T} .{ }^{23}$ Two siblings without cardiac involvement shared c. $-32-13 \mathrm{~T}>\mathrm{G}^{15}$ and c.258dupC. ${ }^{27}$ Three unrelated patients shared c. $-32-13 \mathrm{~T}>\mathrm{G}^{15}$ and c.525delT. ${ }^{13}$

\section{Cardiac history and function}

All patients with IOPD (by definition) and 6 of 26 with LOPD had cardiac involvement. LVM $z$-scores (M-mode) at entry ranged from -4.0 to 10.3 (mean $\pm \mathrm{SD},-0.1 \pm 2.63)[n=85]$ ) overall. Cardiac conditions reported in cardiovascular history at study entry or ascertained by baseline assessment are described below.

\section{IOPD}

A history of cardiomegaly was reported in 72/87 (83\%) IOPD patients at study entry (Supplementary Table S5 lists all IOPD patients' cardiac symptoms and their onset ages). LVM $z$-scores at study entry ranged from -3.8 to 10.3 (mean \pm SD, $0.4 \pm 2.80$ [ $n=62$ evaluable $]$ ) in IOPD. Thirteen (15\%) had cardiomyopathy or hypertrophy without reported cardiomegaly. One patient (1\%) had interventricular septal hypokinesis alone reported at study entry, and one (1\%) had dysrhythmia alone at diagnosis. Many patients' histories of $\mathrm{CHF}$ or dysrhythmias coexisted with cardiomegaly or cardiomyopathy: 26 (30\%) IOPD patients had CHF. Other heart conditions affected 26 (30\%) IOPD patients. Of these, 8 had arrhythmia (unspecified), 7 had Wolff-Parkinson-White (WPW) syndrome (3 without and 4 with supraventricular tachycardia [SVT]), 6 had SVT without WPW, 1 had sinus tachycardia, and 4 had unspecified tachycardia. SVT predominantly affected patients with deletions or nonsense variants.

\section{LOPD}

Six of $26(23 \%)$ LOPD patients had cardiac involvement (Supplementary Table S4). Two of six also had CHF; one had SVT. One patient had SVT and WPW without cardiomegaly, cardiomyopathy, or CHF. LVM $z$-scores for LOPD overall were -4.0 to 1.0 (mean $\pm \mathrm{SD},-1.5 \pm 1.42[n=23]$ ), and among the six cardiac-affected LOPD patients, -1.58 to 0.66 .

\section{Respiratory function}

Baseline seated FVC mean \pm SD was $66.6 \pm 29.6 \%$ predicted in 24 evaluable patients (15 IOPD, $55.9 \pm 26.7 \%$ predicted; 9 LOPD, $84.4 \pm 26.3 \%$ predicted).

\section{Motor function}

GMFM-88 total \% scores were available for 108 patients. Means, medians, and ranges overall and for IOPD and LOPD, along with Pompe motor function categories at study entry, appear in Table 1. Motor decline in the 2 pre-enrollment months was noted in 7 patients $<2$ years old (5 IOPD, 2 LOPD) and 30 patients $\geq 2$ years old ( 24 IOPD, 6 LOPD).

\section{Biochemical markers}

Serum creatine kinase activity $(n=107)$ averaged $1353 \pm 1001$ units (U)/L (median, $1147 \mathrm{U} / \mathrm{L}$; range, 58-5499 U/L; normal, 60-305 U/L $\left.\mathrm{L}^{33}\right)$. Serum aspartate transaminase $(n=103)$ averaged $290 \pm 189 \mathrm{U} / \mathrm{L}$ (median, $257 \mathrm{U} / \mathrm{L}$; range, 16-980 U/L; normal, $\left.15-41 \mathrm{U} / \mathrm{L}^{33}\right)$. Serum alanine transaminase $(n=107)$ averaged $165 \pm 102 \mathrm{U} / \mathrm{L}$ (median, $159 \mathrm{U} / \mathrm{L}$; range, 7-676 U/L; normal, $\left.5-40 \mathrm{U} / \mathrm{L}^{33}\right)$. Serum brain natriuretic peptide $(n=96)$ averaged $31.50 \pm 82.369 \mathrm{pg} / \mathrm{mL}$ (median, 14.62; range, $5.03-784.95 \mathrm{pg} / \mathrm{mL}$; normal, $\left.<20 \mathrm{pg} / \mathrm{mL}^{34}\right)$. Urinary hexose tetrasaccharide was not determined in ADVANCE.

\section{DISCUSSION}

The ADVANCE baseline cohort provides the largest multiethnic, systematically assessed data set to connect Pompe genotypes, phenotypes, and clinical manifestations in the United States.

The phenotypically unrestricted cohort spanned the clinical spectrum of Pompe disease, including IOPD and LOPD. The minority (17\%) of CRIM-negative IOPD patients may reflect fewer surviving to join ADVANCE. Diagnostic and prestudy $160 \mathrm{~L}$ rhGAA initiation ages reflect that some patients had delayed diagnosis and/or extended disease evolution before initiation of treatment.

Cardiac involvement in LOPD is occasionally reported. ${ }^{35}$ Six patients who had initial symptom onsets at $>1$ year (thus LOPD) had cardiac involvement, which often presented later than first symptoms at ages from 1.03 years to $>5$ years (Supplementary Table S4). All six lacked the common Caucasian LOPD splice variant c. $-32-13 \mathrm{~T}>\mathrm{G}$ (IVS1), which typically does not confer cardiac involvement. ${ }^{36}$ These patients had other symptoms (e.g., gastric reflux, sleep apnea, hearing loss) suggesting more severe disease. ${ }^{37}$ Compound heterozygosity for attenuated variants among these patients suggests that severe variants in trans can lead to a more severe 
LOPD phenotype or one intermediate between IOPD and LOPD. For example, exon 18 deletions conferred a cardiac LOPD phenotype when combined with attenuated variants. Participants who developed cardiomyopathy beyond 1 year of age $(\leq 5.3$ years in LOPD), especially those without the common IVS1 splice site variant, illustrate the need for continued cardiac vigilance with increasing age in LOPD. $^{35}$

Mean LVM $z$-score in IOPD was below hypertrophic cardiomyopathy levels at study entry, but SDs and maxima suggest persistent hypertrophy at study entry in some patients. This could reflect short treatment duration before study entry, CRIM-negative status, high-sustained antibody titers at entry, or echocardiographic variability. Overall LOPD mean and maximum LVM $z$-scores were not hypertrophic, and the minimum was below normal. LOPD patients with cardiac histories nevertheless had LVM $z$-scores within normal range at study entry. Potential challenges in echocardiographic data acquisition suggest caution in interpreting score extremes. Diverse prestudy $160 \mathrm{~L}$ rhGAA treatment durations likely increased heterogeneity.

Cardiac rhythm abnormalities among ADVANCE IOPD patients included supraventricular tachycardia, short PR interval, and ST abnormalities, all previously reported in treated IOPD. ${ }^{37}$

Motor histories, including loss of prior ambulation, motor milestone regression, or other motor decline reported during the two prestudy months, demonstrated the tendency of motor gains on alglucosidase alfa to regress with increasing age. $^{37}$ Of note, 34 never-ambulatory patients were $>2$ years old at enrollment (28 IOPD, 6 LOPD), beyond the normal developmental window for walking. This could be related to advanced stage at $160 \mathrm{~L}$ initiation.

Invasive or noninvasive ventilation in $38 \%$ of ADVANCE patients indicates respiratory muscle weakness persisting at study entry. Among the 24 testable patients, mean FVC \% predicted was higher in LOPD than IOPD, reflecting less severe respiratory muscle involvement. One IOPD patient and one LOPD patient had experienced 5-10 pneumonia episodes in the prior year; none experienced $>10$. Overall pneumonia rate $(66 \%)$ exceeded that expected for age. ${ }^{38}$ The small number of patients who were both ventilator-free and old enough for reliable FVC measurement is a limitation.

Growth parameter $z$-scores (Table 1) were within 2 SDs of age norms, as published ${ }^{37}$ for long-term IOPD survivors on $160 \mathrm{~L}$ rhGAA. However, $z$-score minima below -2 indicated residual growth deficits in some ADVANCE participants, possibly related to motor difficulties with feeding. Swallowing impairment and/or gastroesophageal reflux, known in treated IOPD $^{37}$ and LOPD, ${ }^{33}$ were also prevalent in ADVANCE. Nearly $52 \%$ of participants with IOPD and $27 \%$ with LOPD required tube feeding.

ADVANCE findings corroborate multisystem involvements in long-term treated Pompe disease, e.g., hearing loss in treated IOPD $^{37}$ and LOPD. ${ }^{39}$ Histories collected at ADVANCE entry showed conductive loss in $15 \%$ of IOPD and $8 \%$ of LOPD patients. Sensorineural loss affected $7 \%$ of IOPD and $4 \%$ of LOPD patients. In a prior audiometric study, ${ }^{39}$ sensorineural hearing loss affected 10/11 (91\%) IOPD patients but no LOPD patients, and conductive loss affected $1 / 13$ (8\%) LOPD patients.

Apparently unreported GAA variants found in ADVANCE include three in-cis combinations, two splice variants (one in both IOPD and LOPD, the other in IOPD), and one missense variant in IOPD.

The IVS1 splice variant, c. $-32-13 \mathrm{~T}>\mathrm{G}$, is common in Caucasians with LOPD. None of the 12/26 LOPD patients (46\%) in ADVANCE with this variant had cardiac involvement. Conversely, none of six LOPD patients with cardiac involvement had this variant. ADVANCE corroborates Herbert et al., who found occasional arrhythmia and valvular abnormalities but no hypertrophic cardiomyopathy among $<18$-year-olds with the common c. $-32-13 \mathrm{~T}>\mathrm{G}$ variant. ${ }^{36}$ The one c. $-32-13 \mathrm{~T}>\mathrm{G}$ homozygote in ADVANCE had symptom onset at a typical clinically diagnosed age (8.92 years). In contrast, four published newborn-screened c. -32 $-13 \mathrm{~T}>\mathrm{G}$ homozygotes had subtle motor signs by 6 months of age. $^{33}$

In ADVANCE, exon 18 deletions co-occurred with cardiac involvement in five IOPD and two of three LOPD patients bearing them. While exon 18 deletions are associated with IOPD and occur compound heterozygously with less severe variants in LOPD, ${ }^{20}$ their relationship to LOPD cardiac involvement needs further study.

Of the 17 IOPD patients starting $160 \mathrm{~L}$ rhGAA at $>12$ months of age (bold type in Supplementary Table S5), 14 were CRIM-positive, 2 CRIM-negative, and 1 had missing data. The predominantly CRIM-positive status of IOPD patients surviving to treatment initiations at $>1$ year of age (some of whose cardiac involvement occurred later than initial symptom onset) reinforces a spectrum of involvement in IOPD.

ADVANCE contains a diverse population. No complete genotypes were shared between participants with IOPD and LOPD, reinforcing the role of variant types and combinations in determining disease course.

Limitations of ADVANCE baseline data include approximations in onset, diagnosis or $160 \mathrm{~L}$ initiation ages reported historically, particularly for adolescent patients at study entry. Also, retrospective application of IOPD and LOPD definitions revealed some patients as intermediate on the disease spectrum (less severe IOPD with $160 \mathrm{~L}$ initiations $>12$ months of age; cardiac-involved LOPD). Highly variable $160 \mathrm{~L}$ rhGAA treatment durations, possible prestudy regimen variations, and incomplete echocardiographic and pulmonary data are other limitations. The strength of the ADVANCE study is that it represents the largest and most inclusive Pompe disease cohort with systematic and comprehensive assessments in the United States. ADVANCE has expanded the number of GAA pathogenic variants observed and has helped further characterize the phenotypic spectrum of Pompe disease. 


\section{Data sharing}

Qualified researchers may request access to patient-level data and related study documents including the clinical study report, study protocol with any amendments, blank case report form, statistical analysis plan, and data set specifications. Patient-level data will be anonymized and study documents will be redacted to protect the privacy of trial participants. Further details on Sanofi's data sharing criteria, eligible studies, and process for requesting access can be found at https://www.clinicalstudydatarequest.com.

\section{SUPPLEMENTARY INFORMATION}

The online version of this article (https://doi.org/10.1038/s41436019-0527-9) contains supplementary material, which is available to authorized users.

\section{ACKNOWLEDGEMENTS}

This study was supported by Sanofi Genzyme. Medical writing support under the sole scientific control of the authors was provided by Kim Coleman Healy, PhD, CMPP of Envision Medical Affairs (contracted by Sanofi Genzyme). The authors acknowledge Catherine Wilson, DPT, MSPH (contracted by Sanofi Genzyme when the study was conducted) for design, analysis, and interpretation of motor assessments; Robert Pomponio, PhD of Sanofi Genzyme for review and interpretation of genotype data; and additional investigators at the 52 study sites for contributions to patient care and data acquisition. ADVANCE results have been presented in part at WORLDSymposium ${ }^{\mathrm{TM}}, 29$ February-4 March 2016, San Diego, CA, USA; Muscular Dystrophy Association Clinical Meeting, 20-23 March 2016, Arlington, VA, USA; American College of Medical Genetics and Genomics, 8-12 March 2016, Tampa, FL, USA; Society for Inherited Metabolic Disease, 3-6 April 2016, Ponte Vedra Beach, FL, USA; Child Neurology Society, 4-7 October 2017, Kansas City, MO, USA; and American College of Medical Genetics and Genomics, 10-14 April 2018, Charlotte, NC, USA.

\section{DISCLOSURE}

This study was supported by Sanofi Genzyme. Authors employed by Sanofi Genzyme (K.A.H.,S.E.S.; statistician Y.Z. was employed by Sanofi Genzyme when the study was conducted) participated in study design, data collection and interpretation, provided intellectually important revisions to the manuscript, and approved the final version for publication. The authors disclose the following conflicts of interest. From Sanofi Genzyme: S.H.H.: honoraria and travel support (ADVANCE investigators' meeting); D.K.: research funding; N.D.L.: advisory board honoraria, professional writing support; L.D.M.P.: Pompe and Gaucher advisory boards and Gaucher speakers' bureau; P.T.: honoraria, travel support as clinical trial principal investigator; J.B.G.: research support, speakers' bureau; D.W.S.: Pompe Registry Advisory Board, speakers' bureau, research support, honoraria; R.W.: speaking stipend and travel support, 2014 Pompe Registry meeting; J.L.G., grants during the study; P.S.K.: Pompe and Gaucher Registry advisory boards, research support. From Actus Therapeutics: P.S.K., equity. From Alexion, Amicus, Shire, and
Vertex: P.S.K., personal fees. From Baebies, Inc.: P.S.K., advisory board. From Biomarin: P.T., honoraria, travel support as clinical trial principal investigator. From New York Medical College: D.K., research funding. From Roche, Shire, and Valerion: P.S.K., grants. From Seattle Children's Hospital: S.H.H., personal fees (outside submitted work). The other authors declare no conflicts of interest.

Publisher's note: Springer Nature remains neutral with regard to jurisdictional claims in published maps and institutional affiliations.

\section{REFERENCES}

1. Kishnani PS, Beckemeyer AA. New therapeutic approaches for Pompe disease: enzyme replacement therapy and beyond. Pediatr Endocrinol Rev. 2014;12 suppl 1:114-124.

2. Kishnani PS, Amartino HM, Lindberg C, Miller TM, Wilson A, Keutzer J. Timing of diagnosis of patients with Pompe disease: data from the Pompe registry. Am J Med Genet A. 2013;161A:2431-2443.

3. Bali DS, Goldstein JL, Banugaria $S$, et al. Predicting cross-reactive immunological material (CRIM) status in Pompe disease using GAA mutations: lessons learned from 10 years of clinical laboratory testing experience. Am J Med Genet C Semin Med Genet. 2012;160C:40-49.

4. Shimada $Y$, Kobayashi $H$, Kawagoe $S$, et al. Endoplasmic reticulum stress induces autophagy through activation of p38 MAPK in fibroblasts from Pompe disease patients carrying c.546G >T mutation. Mol Genet Metab. 2011;104:566-573.

5. Kishnani PS, Corzo D, Nicolino M, et al. Recombinant human acid [alpha]glucosidase: major clinical benefits in infantile-onset Pompe disease. Neurology. 2007:68:99-109.

6. Nicolino M, Byrne B, Wraith JE, et al. Clinical outcomes after long-term treatment with alglucosidase alfa in infants and children with advanced Pompe disease. Genet Med. 2009;11:210-219.

7. van der Ploeg AT, Clemens PR, Corzo D, et al. A randomized study of alglucosidase alfa in late-onset Pompe's disease. $N$ Engl J Med. 2010;362:1396-1406.

8. Hahn SH, Kronn D, Leslie ND, et al. Efficacy, safety profile, and immunogenicity of alglucosidase alfa produced at the 4,000-liter scale in US children and adolescents with Pompe disease: ADVANCE, a phase IV, open-label, prospective study. Genet Med. 2018;20:1284-1294.

9. World Medical Association Declaration of Helsinki-Ethical Principles for Medical Research Involving Human Subjects (1964, amended 2008 and 2013). https://www.wma.net/policies-post/wma-declaration-of-helsinkiethical-principles-for-medical-research-involving-human-subjects/. Accessed May 7, 2019.

10. van Capelle $\mathrm{Cl}$, van der Beek NA, de Vries JM, et al. The quick motor function test: a new tool to rate clinical severity and motor function in Pompe patients. J Inherit Metab Dis. 2012;35:317-323.

11. Winkel LP, Van den Hout JM, Kamphoven JH, et al. Enzyme replacement therapy in late-onset Pompe's disease: a three-year follow-up. Ann Neurol. 2004;55:495-502.

12. Hermans MM, de Graaff $E$, Kroos MA, et al. The conservative substitution Asp-645-> Glu in lysosomal alpha-glucosidase affects transport and phosphorylation of the enzyme in an adult patient with glycogen-storage disease type II. Biochem J. 1993;289 pt 3:687-693.

13. Hirschhorn R, Huie ML. Frequency of mutations for glycogen storage disease type II in different populations: the delta525T and deltaexon 18 mutations are not generally "common" in white populations. J Med Genet. 1999;36:85-86.

14. Bali DS, Goldstein JL, Rehder C, et al. Clinical laboratory experience of blood CRIM testing in infantile Pompe disease. Mol Genet Metab Rep. 2015;5:76-79

15. Huie $M L$, Chen AS, Tsujino $S$, et al. Aberrant splicing in adult onset glycogen storage disease type II (GSDI): molecular identification of an IVS1 $(-13 T->G)$ mutation in a majority of patients and a novel IVS10 (+1GT->CT) mutation. Hum Mol Genet. 1994;3:2231-2236.

16. Amartino $H$, Painceira $D$, Pomponio RJ, et al. Two clinical forms of glycogen-storage disease type II in two generations of the same family. Clin Genet. 2006;69:187-188. 
17. Erasmus MC Pompe Center. Mutations in human acid alpha-glucosidase. May 2016. http://cluster15.erasmusmc.nl/klgn/pompe/mutations.html? lang=en. Accessed 8 March 2019.

18. Kishnani PS. Duke GAA Mutation Database. June 3, 2014. https:// pediatrics.duke.edu/sites/pediatrics.duke.edu/files/field/attachments/ GAA_mutation_database.pdf. Accessed 8 March 2019.

19. Tan QK, Stockton DW, Pivnick E, et al. Premature pubarche in children with Pompe disease. J Pediatr. 2015;166:1075-18 e1071.

20. Kroos MA, Van der Kraan M, Van Diggelen OP, et al. Glycogen storage disease type II: frequency of three common mutant alleles and their associated clinical phenotypes studied in 121 patients. J Med Genet. 1995;32:836-837.

21. Herzog A, Hartung R, Reuser AJ, et al. A cross-sectional single-centre study on the spectrum of Pompe disease, German patients: molecular analysis of the GAA gene, manifestation and genotype-phenotype correlations. Orphanet J Rare Dis. 2012;7:35.

22. Yang CC, Chien YH, Lee NC, et al. Rapid progressive course of later-onset Pompe disease in Chinese patients. Mol Genet Metab. 2011;104:284-288.

23. Kroos M, Pomponio RJ, van Vliet $L$, et al. Update of the Pompe disease mutation database with 107 sequence variants and a format for severity rating. Hum Mutat. 2008;29:E13-26.

24. Hermans MM, van Leenen D, Kroos MA, et al. Twenty-two novel mutations in the lysosomal alpha-glucosidase gene (GAA) underscore the genotype-phenotype correlation in glycogen storage disease type II. Hum Mutat. 2004;23:47-56.

25. Pittis MG, Montalvo AL, Miocic S, et al. Identification of four novel mutations in the alpha glucosidase gene in five Italian patients with infantile onset glycogen storage disease type II. Am J Med Genet A. 2003; 121A:225-230.

26. Kroos M, Hoogeveen-Westerveld $M$, Michelakakis $H$, et al. Update of the Pompe disease mutation database with 60 novel GAA sequence variants and additional studies on the functional effect of 34 previously reported variants. Hum Mutat. 2012;33:1161-1165.

27. Gort L, Coll MJ, Chabas A. Glycogen storage disease type II in Spanish patients: high frequency of c.1076-1G>C mutation. Mol Genet Metab. 2007;92:183-187.

28. Ko TM, Hwu WL, Lin YW, et al. Molecular genetic study of Pompe disease in Chinese patients in Taiwan. Hum Mutat. 1999;13:380-384.

29. Markic J, Polic B, Stricevic L, et al. Effects of immune modulation therapy in the first Croatian infant diagnosed with Pompe disease: a 3-year follow-up study. Wien Klin Wochenschr. 2014;126:133-137.

30. Elder ME, Nayak S, Collins SW, et al. B-cell depletion and immunomodulation before initiation of enzyme replacement therapy blocks the immune response to acid alpha-glucosidase in infantile-onset Pompe disease. J Pediatr. 2013;163:847-54 e841.
31. Prakalapakorn SG, Proia AD, Yanovitch TL, et al. Ocular and histologic findings in a series of children with infantile pompe disease treated with enzyme replacement therapy. J Pediatr Ophthalmol Strabismus. 2014;51:355-362.

32. Pipo JR, Feng JH, Yamamoto T, et al. New GAA mutations in Japanese patients with GSDII (Pompe disease). Pediatr Neurol. 2003;29: 284-287.

33. Rairikar MV, Case LE, Bailey LA, et al. Insight into the phenotype of infants with Pompe disease identified by newborn screening with the common c.-32-13T>G "late-onset" GAA variant. Mol Genet Metab 2017;122:99-107

34. Angermann CE, Ertl G. [Natriuretic peptides-new diagnostic markers in heart disease]. Herz. 2004;29:609-617.

35. Lee DH, Qiu WJ, Lee J, Chien YH, Hwu WL. Hypertrophic cardiomyopathy in Pompe disease is not limited to the classic infantile-onset phenotype. JIMD Rep. 2014;17:71-75.

36. Herbert M, Cope H, Li JS, Kishnani PS. Severe cardiac involvement is rare in patients with late-onset Pompe disease and the common c.-32-13T>G variant: implications for newborn screening. J Pediatr. 2018;198:308-312.

37. Prater SN, Banugaria SG, DeArmey SM, et al. The emerging phenotype of long-term survivors with infantile Pompe disease. Genet Med. 2012;14:800-810.

38. Denny FW, Clyde WA Jr. Acute lower respiratory tract infections in nonhospitalized children. J Pediatr. 1986;108:635-646.

39. van Capelle $\mathrm{Cl}$, Goedegebure A, Homans NC, Hoeve HL, Reuser AJ, van der Ploeg AT. Hearing loss in Pompe disease revisited: results from a study of 24 children. J Inherit Metab Dis. 2010;33:597-602.

Open Access This article is licensed under a Creative Commons Attribution-NonCommercial-NoDerivatives 4.0 International License, which permits any non-commercial use, sharing, distribution and reproduction in any medium or format, as long as you give appropriate credit to the original author(s) and the source, and provide a link to the Creative Commons license. You do not have permission under this license to share adapted material derived from this article or parts of it. The images or other third party material in this article are included in the article's Creative Commons license, unless indicated otherwise in a credit line to the material. If material is not included in the article's Creative Commons license and your intended use is not permitted by statutory regulation or exceeds the permitted use, you will need to obtain permission directly from the copyright holder. To view a copy of this license, visit http://creativecommons.org/licenses/by-nc-nd/4.0/.

(C) The Author(s) 2019 